\title{
Endurance training lowers ribosome density despite increasing ribosome biogenesis markers in rodent skeletal muscle
}

\author{
Matthew A. Romero ', C. Brooks Mobley ${ }^{1}$, Melissa A. Linden ${ }^{3,4}$, Grace Margaret-Eleanor Meers ${ }^{3,4}$, \\ Jeffrey S. Martin ${ }^{1,2}$, Kaelin C. Young ${ }^{1,2}$, R. Scott Rector ${ }^{3}$ and Michael D. Roberts ${ }^{1,2,5^{*}}$ (D)
}

\begin{abstract}
Objective: The purpose of this study was to examine if: (a) high sugar/high fat Western diet (WD)-feeding affects skeletal muscle ribosome biogenesis markers in hyperphagic, diabetic-prone Otsuka Long-Evans Tokushima Fatty (OLETF) rats, and (b) 12 weeks of treadmill training rescued potential detriments that WD feeding exerted on these markers.
\end{abstract}

Methods: Eight week-old male OLETF rats were fed a low-fat control diet (O-CON, $n=10)$ or high/sucrose/cholesterol Western diet (WD). At weeks 20-32 of age, WD-fed rats were divided into WD sedentary (O-WD/SED, $n=16)$, or WD treadmill trained (5 days/week, $60 \mathrm{~min} /$ day) $(\mathrm{O}-\mathrm{WD} / \mathrm{EX}, \mathrm{n}=10)$ conditions.

Results: Interestingly, total RNA (i.e., ribosome density) was 2.3-fold greater in O-WD/SED versus O-WD/EX rats $(p=0.003)$ despite levels of upstream binding factor protein, RNA polymerase I protein and pre-45S rRNA being greater in O-WD/EX rats. Ribophagy (USP10 and G3BP1) and TRAMP-exosome rRNA degradation pathway (EXOSC10 and SKIV2L2) proteins were assayed to determine if these pathways were involved with lower ribosome density in O-WD/EX rats. While USP10 was higher in O-CON versus O-WD/SED and O-WD/EX rats $(p<0.001$ and $p<0.001$, respectively), G3BP1, EXOSC10 and SKIV2L2 did not differ between groups. Nop56 and Ncl mRNAs, ribosome assembly markers, were highest in O-WD/EX rats. However, Fbl mRNA and $28 \mathrm{~S}$ rRNA, downstream ribosome processing markers, were lowest in O-WD/EX rats. Collectively these data suggest that, in WD-fed rats, endurance training increases select skeletal muscle ribosome biogenesis markers. However, endurance training may reduce muscle ribosome density by interfering with rRNA processing and/or export through mechanisms independent of ribophagy or rRNA degradation.

Keywords: Ribosome biogenesis, Endurance training, Western diet, Ribophagy, rRNA degradation

\section{Introduction}

Type II diabetes (T2D) impairs anabolic signaling in skeletal muscle as shown by Stephens et al. [1] who reported a decrease in eukaryotic initiation factor $4 \mathrm{E}$ binding protein 1 (4EBP1) phosphorylation which, in turn, was associated with decrements in overall muscle protein synthesis. Ribosome biogenesis is a critical regulator

\footnotetext{
${ }^{*}$ Correspondence: mdr0024@auburn.edu

${ }^{5}$ School of Kinesiology, Molecular and Applied Sciences Laboratory, Edward Via College of Osteopathic Medicine-Auburn Campus, Auburn University, 301 Wire Road, Office 286, Auburn, AL 36849, USA

Full list of author information is available at the end of the article
}

of muscle mass given that it catalyzes muscle protein synthesis, and there has been an increased emphasis on investigating the mechanisms that regulate this process [2]. Acute and chronic resistance exercise increases markers of ribosome biogenesis [3, 4]. However, no research has examined how chronic endurance exercise affects the process of ribosome biogenesis. Endurance training improves other facets of skeletal muscle physiology in diabetic rodents (i.e., insulin signaling, mitochondrial biogenesis and microvascular physiology) [5-7]. Thus, it stands to reason that endurance training may also improve markers of ribosome biogenesis in 
diabetic rats which, in turn, facilitates other improvements in skeletal muscle metabolism. Thus, the purpose of this study was to examine if: (a) high sugar/fat/cholesterol (i.e., Western diet) feeding affects various markers of skeletal muscle ribosome biogenesis in hyperphagic, diabetic-prone Otsuka Long-Evans Tokushima Fatty (OLETF) compared to OLETF rats fed a low-fat control diet, and (b) if 12 weeks of endurance training rescued potential detrimental effects that Western diet feeding exerted on skeletal muscle ribosome biogenesis markers (i.e., comparing sedentary and endurance-trained rats fed a Western diet).

\section{Main text \\ Methods}

\section{Animal protocol}

The animal protocol was approved by the Institutional Animal Care and Use Committee at the University of Missouri and complied with the National Institutes of Health's Guide for the Care and Use of Laboratory Animals. Muscle samples assayed in the current study were obtained from male OLETF rats (Tokushima Research Institute, Otsuka Pharmaceutical, Tokushima, Japan) from a prior investigation [8]. Notably, OLETF rats are hyperphagic and become obese hyperglycemic after 18 weeks of age [9]. Briefly, 8-week old OLETF rats were randomized to either consume a control diet $(\mathrm{O}-\mathrm{CON}$, $\mathrm{n}=10$ ) (Diet D12110704, Research Diets Inc.; 10\% kcal fat, $70 \% \mathrm{kcal}$ carbohydrate and $20 \%$ kcal protein, with $3.5 \% \mathrm{kcal}$ sucrose) or Western diet (WD, $\mathrm{n}=26$ ) (Diet D09071604, Research Diets Inc.; 44.9\% kcal fat, $35.1 \% \mathrm{kcal}$ carbohydrate and $20 \% \mathrm{kcal}$ protein, with $1 \%$ weight/weight from cholesterol and $17 \% \mathrm{kcal}$ sucrose) for 24 weeks (up to 32 weeks of age). At 20 weeks of age, the O-WD animals were assigned one of two subset groups which included a sedentary $(\mathrm{O}-\mathrm{WD} / \mathrm{SED}, \mathrm{n}=16)$ or exercise trained $(\mathrm{O}-\mathrm{WD} / \mathrm{EX}, \mathrm{n}=10)$ condition for 12 weeks. Throughout the protocol, all animals were individually housed in standard conditions $(0600 / 1800 \mathrm{~h}$ light/dark cycle at $21^{\circ} \mathrm{C}$ ) and diet and water were provided ad libitum. At 32 weeks of age, animals were overnight fasted and, the morning of dissection, were anaesthetized by intraperitoneal injection of sodium pentobarbital $(100 \mathrm{mg} / \mathrm{kg})$ and were euthanized thereafter by exsanguination. Vastus lateralis muscles were obtained via standard dissection technique, flash frozen in liquid nitrogen and stored at $-80{ }^{\circ} \mathrm{C}$ until analyses described below.

\section{Exercise training for O-WD/EX rats}

At 20 weeks of age, O-WD/EX began treadmill running 5 days/week as described previously [8]. The speed and duration of the treadmill exercise were gradually increased over the first 4 weeks of training until the animals could maintain a running speed of $20 \mathrm{~m} / \mathrm{min}$ for $60 \mathrm{~min} /$ day. By the fifth week of training, animals ran at $20 \mathrm{~m} / \mathrm{min}, 60 \mathrm{~min} /$ day, on a $15 \%$ incline and maintained this until 32 weeks of age. Animals in the O-SED were placed on a non-moving treadmill weekly.

\section{Western blotting procedures}

In-depth Western blotting procedures are similar to what our laboratory have previously published $[3,10]$. Notably, primary antibodies used included the following: (1) rabbit anti-rat RNA polymerase I (RNA Pol I) (1:1000; Thermo Scientific, Rockford, IL, USA), (2) mouse anti-rat upstream binding factor (UBF) (1:1000; Santa Cruz Biotechnology, Dallas, TX, USA), (3) rabbit anti-rat c-myelocytomatosis oncogene (c-Myc) (1:1000; Cell Signaling, Danvers, MA, USA), (4) ubiquitin-specific peptidase 10 (USP10) (1:1000, Cell Signaling), (5) GTPase activating protein binding protein 1 (G3BP1) (1:1000; Santa Cruz Biotechnology, Dallas, TX, USA), (6) mouse anti-rat exosome component 10 (EXOSC10) (1:1000; Santa Cruz Biotechnology, Dallas, TX, USA), (7) mouse anti-rat Superkiller Viralicidic Activity 2-Like 2 (SKIV2L2) (1:1000; Santa Cruz Biotechnology, Dallas, TX, USA).

\section{Total RNA determination and real-time $P C R$}

In-depth total RNA isolation/quantification and realtime PCR methods utilized are similar to what our laboratory have previously published $[3,10]$, and details regarding PCR primers as well as fold-change calculations have been previously published [3]. Of note, cyclophilin A was used as a housekeeping gene for fold-change calculations given that it remained stable across all diet and activity treatments.

\section{Subcellular protein determination}

In-depth protein isolation/quantification methods utilized are similar to what our laboratory have previously published [10]. Due to limited tissue, only a subset of animals were able to be assayed per group $(\mathrm{O}-\mathrm{CON} \mathrm{n}=7$, $\mathrm{O}-\mathrm{WD} / \mathrm{SED} \mathrm{n}=14, \mathrm{O}-\mathrm{WD} / \mathrm{EX} \mathrm{n}=8$ ).

\section{Statistical analyses}

All data are presented in figures as mean \pm standard deviation (SD) values. Statistics were performed using SPSS v22.0 (IBM, Armonk, NY, USA). All dependent variables were compared between treatments using oneway ANOVAs with post hoc independent $t$ test with Bonferroni correction being performed when ANOVA $p$ values were $<0.05$. The partial eta squared statistic $\left(\eta^{2}\right)$ 
was calculated for effect size for all ANOVAs, and values between 0.010 and 0.059 , values between 0.060 and 0.138 and values greater than 0.138 can be interpreted as small, medium, and large effect sizes, respectively. Likewise, 95\% confidence intervals are presented for all dependent variables.

\section{Results}

Body mass, food consumption, serum glucose, serum insulin and homeostatic model assessment of insulin resistance (HOMA-IR) values from each group are presented in Table 1 with accompanying ANOVA $\mathrm{p}$ values, effect sizes, and 95\% confidence intervals. Note that these values are partial $\mathrm{n}$-sizes of data previously presented by Linden et al. [8], and serve to provide information regarding the phenotype of each group for convenience to the reader. Body mass was greater in $\mathrm{O}-\mathrm{WD} / \mathrm{SED}$ versus $\mathrm{O}-\mathrm{CON}(\mathrm{p}<0.05)$, caloric consumption during weeks 20-32 was greater in O-WD/

Table 1 Body mass, food consumption and serum glucose and insulin values

\begin{tabular}{|c|c|c|c|}
\hline Variable & Values (mean \pm SD) & $95 \% \mathrm{Cl}$ & $\begin{array}{l}\text { ANOVA p value (eta- } \\
\text { squared) }\end{array}$ \\
\hline \multicolumn{4}{|c|}{ Sacrifice body mass (g) } \\
\hline O-CON & $648 \pm 80^{\mathrm{a}}$ & $594-718$ & 0.032 \\
\hline O-WD/SED & $726 \pm 87^{b}$ & $686-787$ & $(0.188)$ \\
\hline O-WD/EX & $720 \pm 36^{a, b}$ & $696-744$ & \\
\hline \multicolumn{4}{|c|}{ Kcal consumed (week 20-32) } \\
\hline O-CON & $746 \pm 56^{a}$ & $682-768$ & $<0.001$ \\
\hline O-WD/SED & $843 \pm 39^{b}$ & $803-848$ & $(0.535)$ \\
\hline O-WD/EX & $758 \pm 31^{\mathrm{a}}$ & $736-780$ & \\
\hline \multicolumn{4}{|c|}{ Glucose (mg/dL) } \\
\hline O-CON & $339 \pm 40^{a}$ & $306-371$ & 0.001 \\
\hline O-WD/SED & $341 \pm 65^{a}$ & $303-338$ & $(0.364)$ \\
\hline O-WD/EX & $253 \pm 44^{b}$ & $222-284$ & \\
\hline \multicolumn{4}{|l|}{ Insulin (ng/dL) } \\
\hline O-CON & $5.1 \pm 3.2$ & $2.9-7.9$ & 0.11 \\
\hline O-WD/SED & $3.4 \pm 2.6$ & $2.2-5.2$ & $(0.124)$ \\
\hline O-WD/EX & $2.7 \pm 1.2$ & $1.9-3.6$ & \\
\hline \multicolumn{4}{|l|}{ HOMA-IR } \\
\hline O-CON & $4.4 \pm 3.1^{\mathrm{a}}$ & $2.3-7.1$ & 0.023 \\
\hline O-WD/SED & $2.8 \pm 2.1^{\mathrm{a}, \mathrm{b}}$ & $1.7-4.2$ & $(0.205)$ \\
\hline O-WD/EX & $1.7 \pm 0.7^{b}$ & $1.2-2.1$ & \\
\hline
\end{tabular}

These values are partial $\mathrm{n}$-sizes of data previously presented by Linden et al. [15], and serve to provide information regarding the phenotype of each group for convenience to the reader. All data are presented as mean $\pm S D(n=10-16$ rats per group), $95 \%$ confidence intervals (CI) are presented, and data with different superscript letters indicate between-treatment differences $(p<0.05)$

O-CON OLETF rats consuming a control diet during weeks 20-32 and were not treadmill-trained, O-WD/SED OLETF rats consuming a Western diet during weeks 20-32 and were not treadmill-trained, O-WD/EX OLETF rats consuming a Western diet during weeks 20-32 and were treadmill-trained 5 days/week; HOMA-IR, homeostatic model assessment of insulin resistance
SED versus other groups $(\mathrm{p}<0.05)$, and fasted serum glucose was lowest in O-WD/EX versus other groups $(\mathrm{p}<0.05)$. HOMA-IR values were significantly greater in $\mathrm{O}-\mathrm{CON}$ versus $\mathrm{O}-\mathrm{WD} / \mathrm{EX}$ rats $(\mathrm{p}=0.021)$, suggesting that $\mathrm{O}-\mathrm{CON}$ rats presented a worsened degree of insulin resistance compared to O-WD/SED rats. While HOMA-IR values were $67 \%$ greater in O-WD/SED versus $\mathrm{O}-\mathrm{WD} / \mathrm{EX}$ rats, this failed to reach statistical significance $(\mathrm{p}=0.123)$.

Total RNA (ANOVA $\mathrm{p}=0.010, \eta^{2}=0.260$ ) was significantly greater in the O-WD/SED than the O-WD/EX rats $(\mathrm{p}=0.003$ ) (Fig. 1a). Myofibrillar protein (ANOVA $p=0.260, \eta^{2}=0.092$; Fig. 1b) and muscle soluble protein (ANOVA $\mathrm{p}=0.547, \eta^{2}=0.040$; Fig. 1c) was not different between groups. The ratio of myofibrillar protein to total RNA (ANOVA $\mathrm{p}=0.024, \eta^{2}=0.278$ ), a gross marker of translational efficiency [11], was significantly greater in $\mathrm{O}-\mathrm{WD} / \mathrm{EX}$ versus $\mathrm{O}-\mathrm{WD} / \mathrm{SED}$ rats $(\mathrm{p}=0.040)$, and tended to be greater in $\mathrm{O}-\mathrm{WD} / \mathrm{EX}$ versus $\mathrm{O}-\mathrm{CON}$ rats $(\mathrm{p}=0.061)$ (Fig. $1 \mathrm{~d})$. The ratio of muscle soluble protein to total RNA (ANOVA $\mathrm{p}=0.032$, $\eta^{2}=0.226$ ), another gross marker of translational efficiency $[11,12]$, was significantly greater in O-WD/EX compared to O-WD/SED ( $\mathrm{p}=0.031)$, but was not different between O-CON and O-WD/EX rats $(\mathrm{p}=0.167)$ (Fig. 1e).

c-Myc protein levels (ANOVA $\mathrm{p}=0.019, \eta^{2}=0.219$ ) were significantly greater in O-CON compared to O-WD/SED $(\mathrm{p}=0.030)$, but not $\mathrm{O}-\mathrm{WD} / \mathrm{EX}$ rats (Fig. $2 \mathrm{a})$. UBF protein levels (ANOVA $\mathrm{p}=0.011, \eta^{2}=0.259$ ) were significantly greater in O-WD/EX versus $\mathrm{O}-\mathrm{CON}$ rats $(\mathrm{p}=0.012)$, but not different from $\mathrm{O}-\mathrm{WD} / \mathrm{SED}$ rats (Fig. 2b). RNA Pol I protein levels (ANOVA p $<0.001$, $\left.\eta^{2}=0.383\right)$ were significantly greater in O-WD/EX versus $\mathrm{O}-\mathrm{WD} / \mathrm{SED}$ rats $(\mathrm{p}<0.001)$, and tended to be higher in the former group versus $\mathrm{O}-\mathrm{CON}$ rats $(\mathrm{p}=0.061)$ (Fig. 2c). Pre-45S rRNA (ANOVA $\mathrm{p}=0.001, \eta^{2}=0.360$ ) was significantly greater in $\mathrm{O}-\mathrm{WD} / \mathrm{EX}$ versus $\mathrm{O}-\mathrm{WD} /$ SED and $O-C O N$ rats $(p=0.001$ and $p=0.034$, respectively) (Fig. 2d).

USP10 protein levels (ANOVA $\mathrm{p}<0.001, \eta^{2}=0.675$ ) were significantly greater in $\mathrm{O}-\mathrm{CON}$ versus $\mathrm{O}-\mathrm{WD} / \mathrm{SED}$ and $\mathrm{O}-\mathrm{WD} / \mathrm{EX}$ rats $(\mathrm{p}<0.001$ and $\mathrm{p}<0.001$, respectively) (Fig. 3a), while protein levels of G3BP1 (ANOVA $\mathrm{p}=0.431, \eta^{2}=0.508$; Fig. $3 \mathrm{~b}$ ) EXOSC10 (ANOVA $\mathrm{p}=0.385, \eta^{2}=0.056$; Fig. $3 \mathrm{c}$ ) or SKIV2L2 (ANOVA $\mathrm{p}=0.908, \eta^{2}=0.006$; Fig. 3d) were not different between groups.

Nop56 mRNA (ANOVA $\mathrm{p}<0.001, \mathrm{\eta}^{2}=0.508$ ) was significantly greater in $\mathrm{O}-\mathrm{WD} / \mathrm{EX}$ versus $\mathrm{O}-\mathrm{CON}$ and $\mathrm{O}-\mathrm{WD} / \mathrm{SED}$ rats $(\mathrm{p}=0.024$ and $\mathrm{p}<0.001$, respectively), as well as $\mathrm{O}-\mathrm{CON}$ versus $\mathrm{O}-\mathrm{WD} / \mathrm{SED}$ rats $(\mathrm{p}=0.034)$ (Fig. 4a). Ncl mRNA (ANOVA $\mathrm{p}<0.001, \eta^{2}=0.588$ ) 

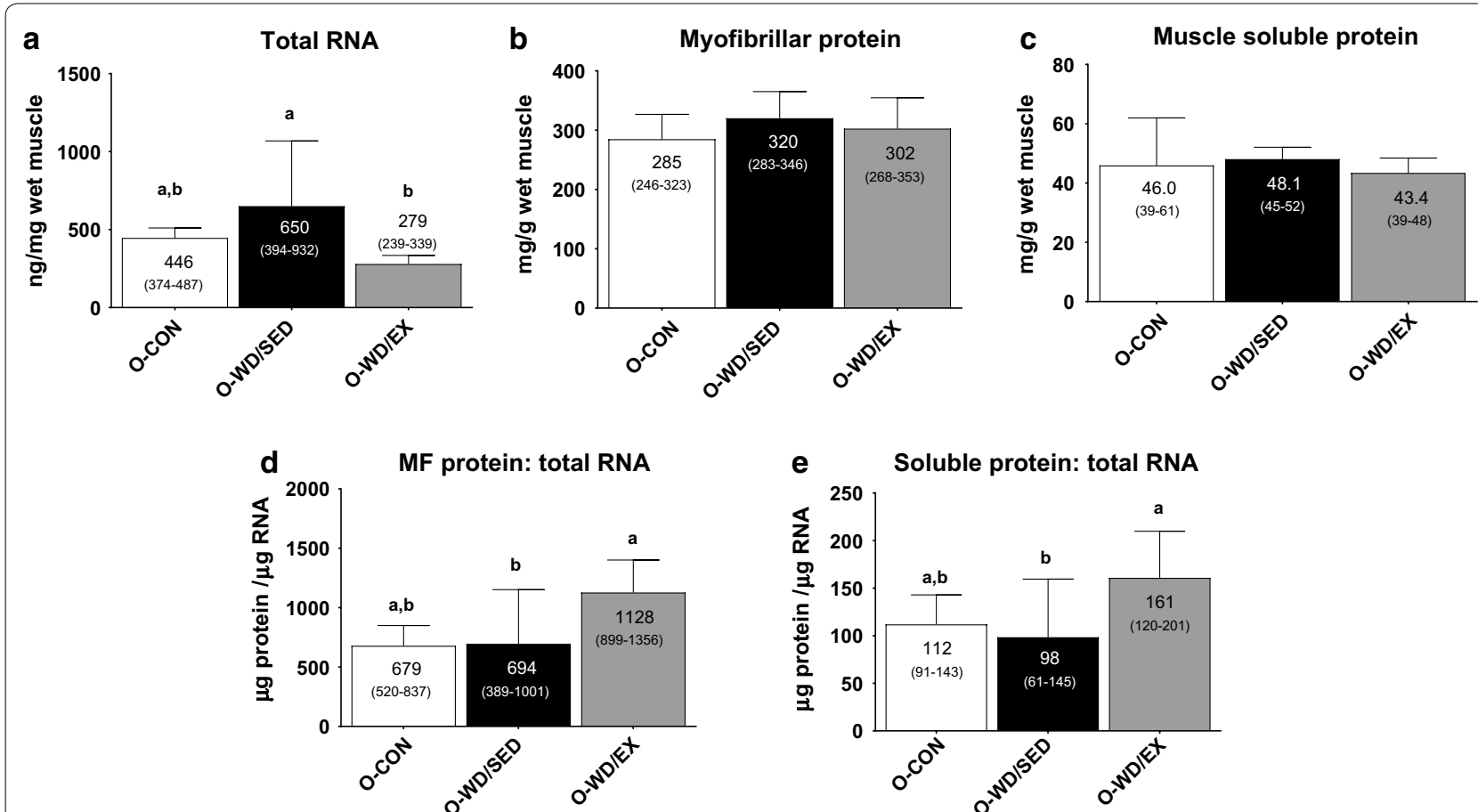

Fig. 1 Effects of diet and exercise on markers of total ribosome content and function. The effects of the intervention on skeletal muscle total RNA levels (i.e., ribosome content) (a), myofibrillar protein levels (b), muscle soluble protein levels (c), myofibrillar (MF): total RNA ratio as a surrogate of ribosome function (d) and muscle soluble protein: total RNA ratio as an additional surrogate of ribosome function (e). All data are presented as mean $\pm S D$ ( $n=10-16$ rats per group), group mean values are presented within each bar, 95\% confidence intervals are presented in parentheses within each bar, and bars with different superscript letters indicate between-treatment differences $(p<0.05)$. Due to limited tissue, only a subset of animals were assayed for myofibrillar protein (O-CON $n=7,0-W D / S E D n=14, O-W D / E X n=8)$. O-CON OLETF rats consuming a control diet during weeks 20-32 and were not treadmill-trained, O-WD/SED OLETF rats consuming a Western diet during weeks 20-32 and were not treadmill-trained, O-WD/EX OLETF rats consuming a Western diet during weeks 20-32 and were treadmill-trained 5 days/week

was significantly greater in $\mathrm{O}-\mathrm{WD} / \mathrm{EX}$ versus $\mathrm{O}-\mathrm{CON}$ and $\mathrm{O}-\mathrm{WD} / \mathrm{SED}$ rats $(\mathrm{p}=0.011$ and $\mathrm{p}<0.001$, respectively), as well as O-CON versus O-WD/SED rats $(\mathrm{p}=0.009$ ) (Fig. 4b). Npm1 mRNA (ANOVA $\mathrm{p}=0.024$, $\left.\eta^{2}=0.216\right)$ was significantly greater in $\mathrm{O}-\mathrm{CON}$ versus $\mathrm{O}-\mathrm{WD} / \mathrm{EX}$ rats $(\mathrm{p}=0.033)$ (Fig. 4c). Fbl mRNA (ANOVA $\left.\mathrm{p}=0.001, \mathrm{\eta}^{2}=0.347\right)$ was significantly greater in O-CON $(\mathrm{p}=0.002)$ and $\mathrm{O}-\mathrm{WD} / \mathrm{SED}(\mathrm{p}=0.004)$ versus $\mathrm{O}-\mathrm{WD} / \mathrm{EX}$ rats (Fig. 4d). 28S rRNA (ANOVA $\mathrm{p}=0.023$, $\left.\eta^{2}=0.223\right)$ was significantly lower in O-WD/EX versus $\mathrm{O}-\mathrm{WD} / \mathrm{SED}$ rats $(\mathrm{p}=0.04)$ (Fig. 4e). There were no between-group differences in 18S rRNA (ANOVA $\left.\mathrm{p}=0.316, \mathrm{\eta}^{2}=0.074\right)$.

\section{Discussion}

Two prior studies $[13,14]$ have reported that skeletal muscle ribosome density was lower in diabetic animals compared to control animals, suggesting that the severity of diabetes and/or insulin resistance may be associated with reduced ribosome density. However, we observed a low ribosome density (total RNA) in O-WD/EX rats despite these rats presenting significantly lower HOMAIR scores compared to the O-CON rats and lower (albeit non-significant) HOMA-IR scores compared to O-WD/ SED rats (Table 1). Therefore, these results are suggestive of an endurance exercise-induced decrease in ribosome density in endurance-trained OLETF rats despite a lower severity of insulin resistance compared to sedentary control and WD-fed rats.

Although investigations have reported that skeletal muscle ribosome function (i.e., muscle protein synthesis) increases acutely with endurance exercise $[15,16]$, no one has examined whether chronic endurance training affects ribosome content and/or markers of ribosome biogenesis and turnover in skeletal muscle. Compared to one or both of the sedentary rat groups $(\mathrm{O}-\mathrm{CON}$ and $\mathrm{O}-\mathrm{WD} /$ SED), O-WD/EX rats presented with: (a) the greatest levels of UBF protein, RNA Pol I protein and pre-45S rRNA 

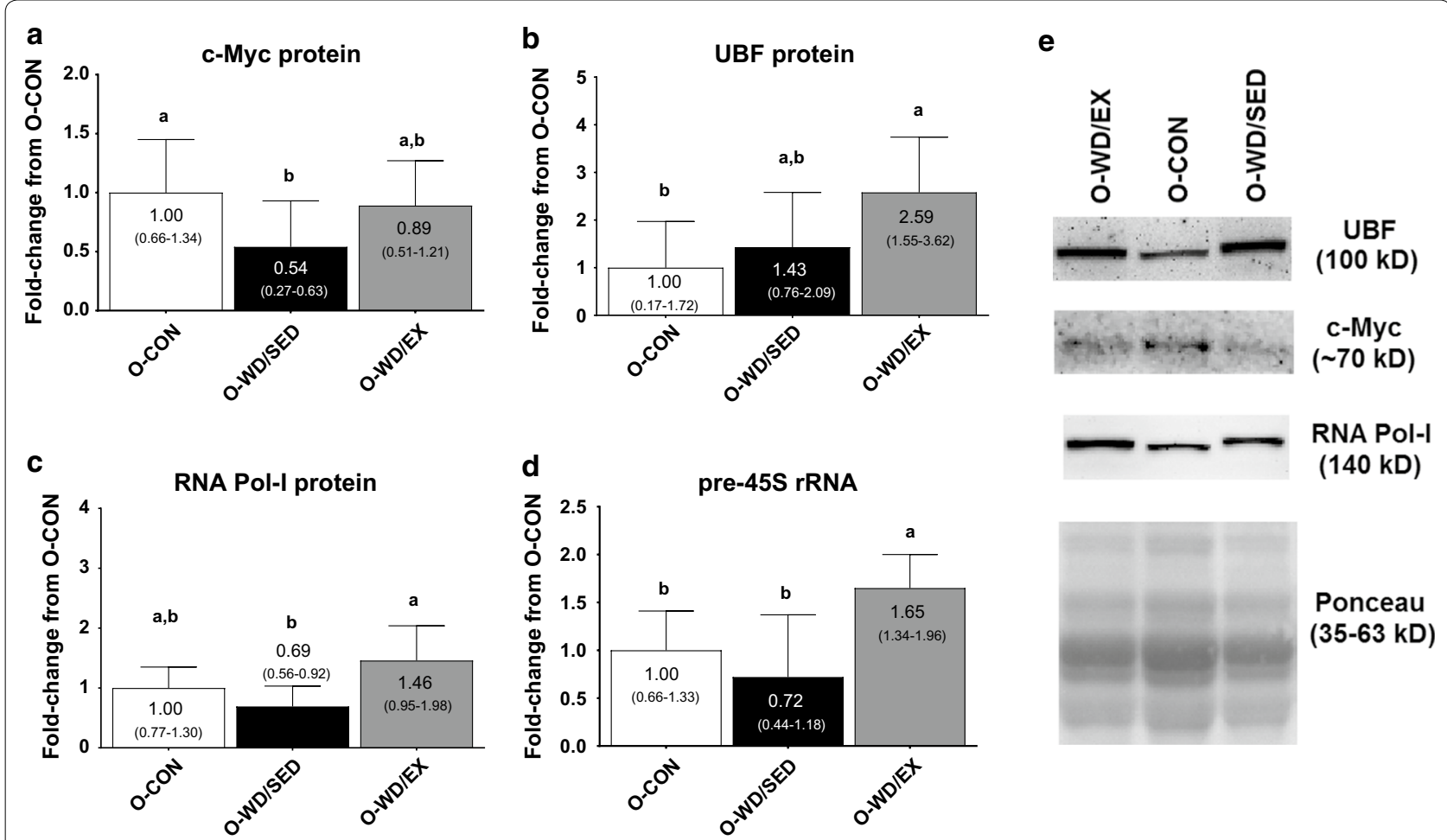

Fig. 2 Effects of diet and exercise on markers of ribosome biogenesis. The effects of the intervention on c-Myc protein levels (a), UBF protein levels (b), RNA Pol I protein levels (c) and pre-45S rRNA transcript levels (d). e Representative images of Western blotting targets. All data are presented as mean $\pm S D$ ( $n=10-16$ rats per group), group mean values are presented within each bar, 95\% confidence intervals are presented in parentheses within each bar, and bars with different superscript letters indicate between-treatment differences $(p<0.05)$. O-CON OLETF rats consuming a control diet during weeks 20-32 and were not treadmill-trained, O-WD/SED OLETF rats consuming a Western diet during weeks 20-32 and were not treadmill-trained, O-WD/EX OLETF rats consuming a Western diet during weeks 20-32 and were treadmill-trained 5 days/week

which is suggestive of increased ribosome biogenesis, (b) the lowest levels of total RNA, and (c) higher expression of select mRNAs related to ribosome processing and formation (i.e., $\mathrm{Ncl}$ and Nop56).

We next assayed ribophagy and TRAMP-exosome pathway targets given that ribosome density was lower in endurance exercised rats. Ribophagy is a selective process in which the autophagy machinery selectively targets mature ribosomes for degradation [17, 18], and involves a protease complex that consists of USP10 and its associated protein G3BP1. The complex acts to deubiquitylate the $60 \mathrm{~S}$ ribosomal subunit and signals the autophagasome to engulf the subunit for degradation [18]. The TRAMP-exosome pathway involves rRNA degradation through intracellular exosomes whereby rRNA is degraded via the exosome's $3^{\prime} \rightarrow 5^{\prime}$ exonuclease activity [19]. Interestingly, skeletal muscle USP10 protein robustly decreased in both the O-WD/SED and O-WD/ EX compared to O-CON rats suggesting that WD feeding downregulates the expression of this ribophagy enzyme; notably, neither G3BP1 nor TRAMP exosome pathway markers were significantly different between groups. We next interrogated the mRNA expression of genes related to rRNA processing (Nop56), ribosome assembly ( $\mathrm{Ncl}$ ), ribosome processing from the pre-45S rRNA transcript (28S rRNA and 18S rRNA) and nuclear ribosome export $(\mathrm{Npm} 1$ and $\mathrm{Fbl})$ in order to examine if these targets were lower in exercised rats. Similar to UBF protein, RNA pol I protein and pre-45S rRNA expression patterns, the expression of select genes involved with rRNA processing and ribosome assembly (Nop56 and $\mathrm{Ncl}$ ) were greatest in exercised rats. Hence, in the WD rats studied herein, endurance training increases certain markers of ribosome biogenesis as well as certain mRNAs related to downstream ribosome processing. However, Npm1 and Fbl mRNA (genes related to nuclear ribosome export) and 28S rRNA levels (indicative of rRNA processing from the pre-45S transcript) were lowest in the O-WD/ 


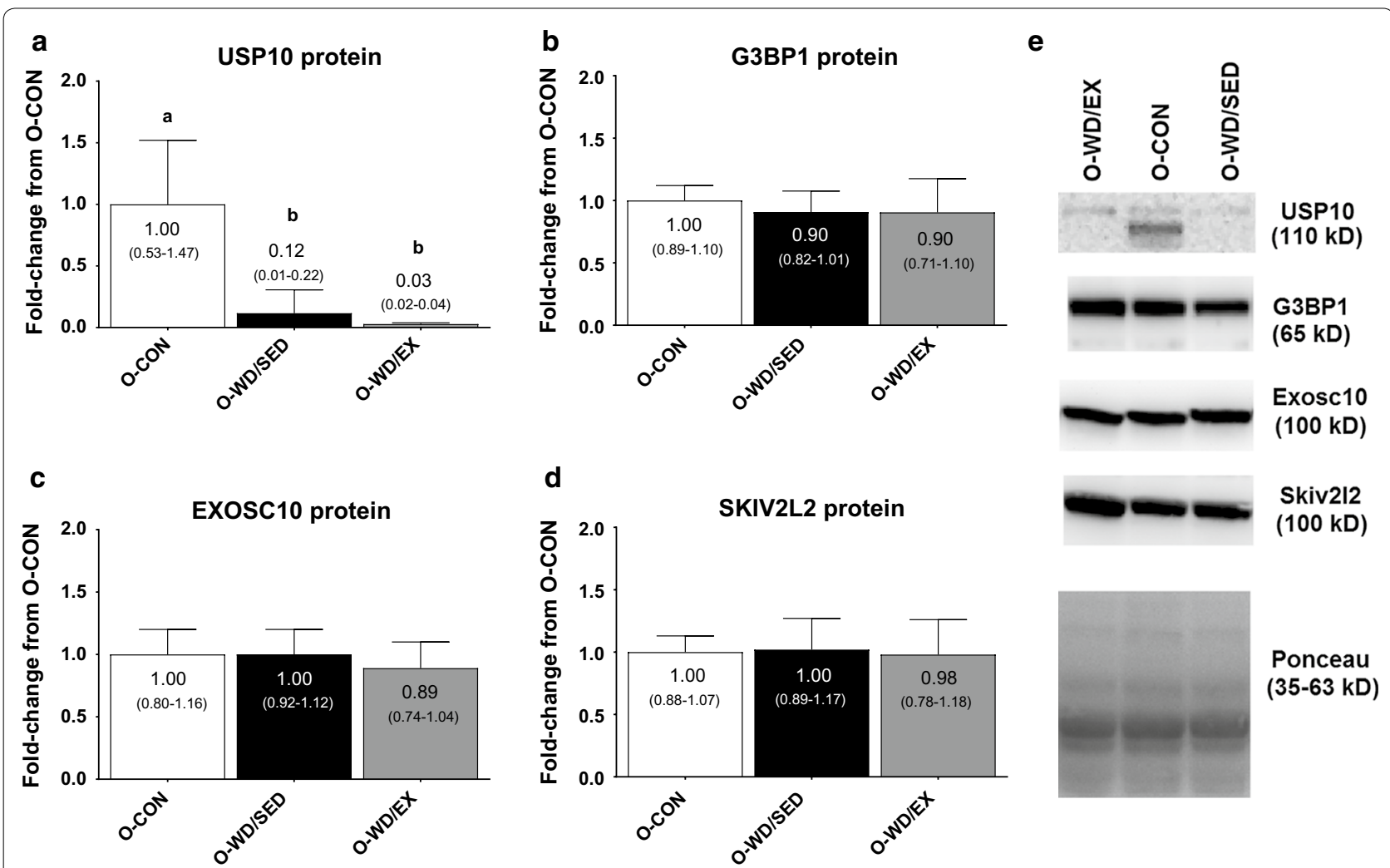

Fig. 3 Effects of diet and exercise on markers of ribosome degradation. The effects of the intervention on USP10 protein levels (a) and G3BP1 protein levels (b) (both markers being critical for ribophagy), as well as EXOSC10 protein levels (c) and SKIV2L2 protein levels (d) (both markers being the TRAMP exosome rRNA degradation pathway). e Representative images of Western blotting targets. All data are presented as mean \pm SD ( $n=10-16$ rats per group), group mean values are presented within each bar, 95\% confidence intervals are presented in parentheses within each bar, and bars with different superscript letters indicate between-treatment differences $(p<0.05)$. O-CON OLETF rats consuming a control diet during weeks 20-32 and were not treadmill-trained, O-WD/SED OLETF rats consuming a Western diet during weeks 20-32 and were not treadmill-trained, O-WD/EX OLETF rats consuming a Western diet during weeks 20-32 and were treadmill-trained 5 days/week

EX group. Therefore, endurance exercise may interfere with rRNA processing and ribosome export through an unidentified mechanism that was not related to ribosome turnover via ribophagy and/or the TRAMP-exosome pathway.

While non-exercised rats exhibited a greater skeletal muscle ribosome density, gross markers of ribosome function were greater in endurance-trained rats. In this regard, $\mathrm{O}-\mathrm{WD} / \mathrm{EX}$ rats presented with the greatest levels of myofibrillar protein to RNA and soluble muscle protein to RNA ratios which is suggestive of increased ribosome function; this finding being in accordance reports suggesting that endurance exercise increases myofibril and mitochondrial protein synthesis rates in rodents [20]. Thus, while endurance exercise may reduce ribosome formation, it does not seem to impair ribosome function in OLETF rats given that myofibril and total soluble muscle protein was not reduced with endurance training.

\section{Limitations}

First, only one sacrificial time point was assessed which precludes us from determining time course mechanisms as to how the assayed markers were altered throughout the intervention. Second, we did not include a nonOLETF control strain in the current study (i.e., leaner LETO rats), so comparing the assayed markers between diabetic-prone OLETF and LETO rats in future studies would yield intuitive information regarding how ribosome biogenesis is affected between strains. Likewise, we did not include an O-CON/EX group which could have provided more in-depth information regarding how both diets with and without exercise affected the markers assayed herein. Notwithstanding, these data provide 


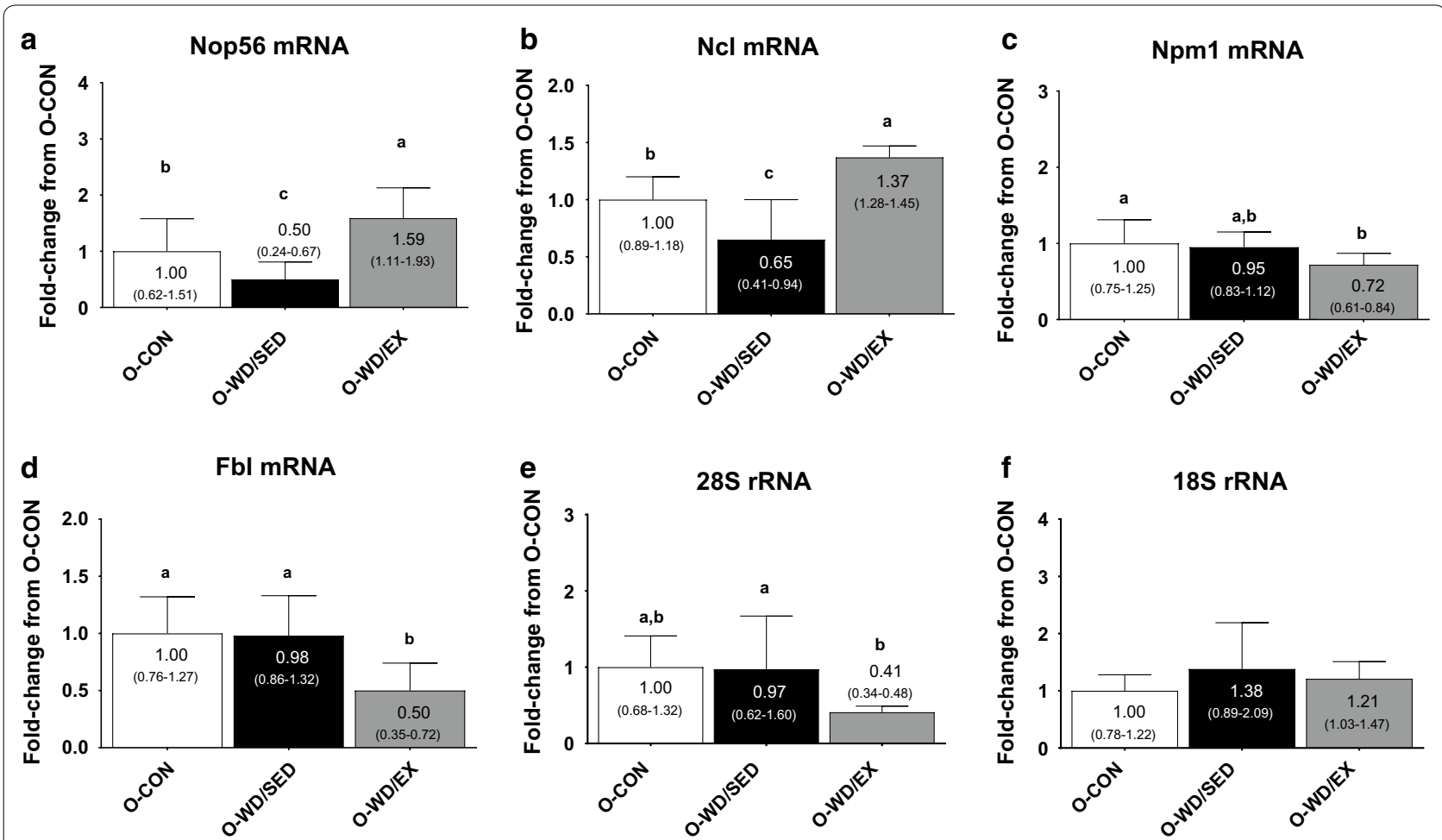

Fig. 4 Effects of diet and exercise on the expression of genes associated with rRNA processing and ribosome export. The effects of the intervention on Nop56 mRNA levels (a) and Ncl mRNA levels (b) (both genes being involved in rRNA processing and ribosome assembly), Npm1 mRNA levels (c) and Fbl mRNA levels (d) (genes involved with nuclear ribosome export), and 285 rRNA transcript levels (e) and 18S rRNA transcript levels (f) (both markers being indicative of downstream rRNA processing from the pre-45S transcript). All data are presented as mean \pm SD ( $n=10-16$ rats per group), group mean values are presented within each bar, 95\% confidence intervals are presented in parentheses within each bar, and bars with different superscript letters indicate between-treatment differences $(p<0.05)$. O-CON OLETF rats consuming a control diet during weeks 20-32 and were not treadmill-trained, O-WD/SED OLETF rats consuming a Western diet during weeks 20-32 and were not treadmill-trained, O-WD/EX OLETF rats consuming a Western diet during weeks 20-32 and were treadmill-trained 5 days/week

novel evidence examining how chronic endurance exercise affects processes related to skeletal muscle ribosome dynamics, and therefore, warrants future work in humans to confirm these findings.

\section{Abbreviations}

4EBP1: eukaryotic initiation factor 4E binding protein 1; ANOVA: analysis of variance; c-Myc: c-myelocytomatosis oncogene; EX: exercise trained; EXOSC10: exosome component 10; Fbl: fibrillarin; G3BP1: GTPase activating protein binding protein 1; HKG: housekeeping gene; HOMA-IR: homeostatic model assessment of insulin resistance; Ncl: nucleolin; Npm1: nucleophosmin; OLETF: Otsuka Long-Evans Tokushima Fatty; Poll/III: RNA polymerase I/III; rRNA: ribosomal ribonucleic acid; SED: sedentary; SKIV2L2: Superkiller Viralicidic Activity 2-Like 2; T2D: type II diabetes; UBF: upstream binding factor; USP10: ubiquitinspecific peptidase 10; WD: Western diet.

\section{Authors' contributions}

MAR: assisted with drafting manuscript, performed statistical analysis and primarily performed all muscle analyses; CBM: assisted with drafting manuscript and critically assisted with all muscle analyses; MAL and GMEM: performed animal husbandry and assisted with drafting manuscript and data interpretation; JSM and KCY: assisted with drafting manuscript and data interpretation; RSR: provided animal tissues, assisted with drafting manuscript and data interpretation; MDR: primarily drafted manuscript and provided intellectual direction and funding for all muscle analyses. All authors read and approved the final manuscript.

\section{Author details}

${ }^{1}$ School of Kinesiology, Auburn University, Auburn, AL, USA. ${ }^{2}$ Department of Cell Biology and Physiology, Edward Via College of Osteopathic MedicineAuburn Campus, Auburn, AL, USA. ${ }^{3}$ Medicine-Division of Gastroenterology and Hepatology, and Nutrition and Exercise Physiology, University of Missouri, Columbia, MO, USA. ${ }^{4}$ Research Service, Harry STruman Memorial VA Hospital, Columbia, MO, USA. ${ }^{5}$ School of Kinesiology, Molecular and Applied Sciences Laboratory, Edward Via College of Osteopathic Medicine-Auburn Campus, Auburn University, 301 Wire Road, Office 286, Auburn, AL 36849, USA.

\section{Acknowledgements}

We thank Romil Patel and Wesley Kephart for critical assistance with Western blotting.

\section{Competing interests}

None of the authors carry professional relationships that could be perceived as a conflict of interest regarding the publication of these data. The results of the study are presented clearly, honestly, and without fabrication, falsification, or inappropriate data manipulation.

\section{Availability of data}

Raw data will be made available upon request. Please email the corresponding author at mdr0024@auburn.edu. 


\section{Consent for publication}

All co-authors have read this manuscript and agree to publish its contents.

\section{Ethical approval and consent to participate}

All procedures involving animals were in accordance with the ethical standards of University of Missouri and procedures were approved by the Animal Care and Use Committee.

Not applicable for human participation given that this was an animal study. As stated prior, all procedures involving animals were in accordance with the ethical standards of University of Missouri and procedures were approved by the Animal Care and Use Committee.

\section{Funding}

This work was partially supported by a Veteran's Health Affair Grant (VHACDA2 1299-01) and University of Missouri Research Council Grant awarded to R. S. Rector. This work was also supported with resources and the use of facilities at the Harry S. Truman Memorial Veterans Hospital in Columbia, MO. All assays presented herein were performed at Auburn University and were supported by laboratory start-up funds given to M. D. Roberts.

\section{Publisher's Note}

Springer Nature remains neutral with regard to jurisdictional claims in published maps and institutional affiliations.

Received: 20 April 2017 Accepted: 6 August 2017

Published online: 11 August 2017

\section{References}

1. Stephens FB, Chee C, Wall BT, Murton AJ, Shannon CE, van Loon LJ, Tsintzas K. Lipid-induced insulin resistance is associated with an impaired skeletal muscle protein synthetic response to amino acid ingestion in healthy young men. Diabetes. 2015;64(5):1615-20.

2. Chaillou T, Kirby TJ, McCarthy JJ. Ribosome biogenesis: emerging evidence for a central role in the regulation of skeletal muscle mass. J Cell Physiol. 2014;229(11):1584-94.

3. Mobley CB, Fox CD, Thompson RM, Healy JC, Santucci V, Kephart WC, McCloskey AE, Kim M, Pascoe DD, Martin JS, et al. Comparative effects of whey protein versus L-leucine on skeletal muscle protein synthesis and markers of ribosome biogenesis following resistance exercise. Amino Acids. 2016:48(3):733-50.

4. West DW, Baehr LM, Marcotte GR, Chason CM, Tolento L, Gomes AV, Bodine SC, Baar K. Acute resistance exercise activates rapamycin-sensitive and -insensitive mechanisms that control translational activity and capacity in skeletal muscle. J Physiol. 2016;594(2):453-68.

5. Castorena CM, Arias EB, Sharma N, Cartee GD. Postexercise improvement in insulin-stimulated glucose uptake occurs concomitant with greater AS160 phosphorylation in muscle from normal and insulin-resistant rats. Diabetes. 2014;63(7):2297-308.
6. Drake JC, Wilson RJ, Yan Z. Molecular mechanisms for mitochondrial adaptation to exercise training in skeletal muscle. FASEB J. 2016;30(1):13-22.

7. Martin JS, Padilla J, Jenkins NT, Crissey JM, Bender SB, Rector RS, Thyfault JP, Laughlin $\mathrm{MH}$. Functional adaptations in the skeletal muscle microvasculature to endurance and interval sprint training in the type 2 diabetic OLETF rat. J Appl Physiol (1985). 2012;113(8):1223-32.

8. Linden MA, Sheldon RD, Meers GM, Ortinau LC, Morris EM, Booth FW, Kanaley JA, Vieira-Potter VJ, Sowers JR, Ibdah JA, et al. Aerobic exercise training in the treatment of non-alcoholic fatty liver disease related fibrosis. J Physiol. 2016;594(18):5271-84.

9. Kawano K, Hirashima T, Mori S, Natori T. OLETF (Otsuka Long-Evans Tokushima Fatty) rat: a new NIDDM rat strain. Diabetes Res Clin Pract. 1994;24(Suppl):S317-20.

10. Mobley CB, Mumford PW, Kephart WC, Conover CF, Beggs LA, Balaez A, Yarrow JF, Borst SE, Beck DT, Roberts MD. Effects of testosterone treatment on markers of skeletal muscle ribosome biogenesis. Andrologia. 2016:48(9):967-77.

11. Adams GR, Haddad F. The relationships among IGF-1, DNA content, and protein accumulation during skeletal muscle hypertrophy. J Appl Physiol (1985). 1996;81(6):2509-16.

12. Roberts MD, Kerksick CM, Dalbo VJ, Hassell SE, Tucker PS, Brown R. Molecular attributes of human skeletal muscle at rest and after unaccustomed exercise: an age comparison. J Strength Cond Res. 2010;24(5):1161-8.

13. Flaim KE, Copenhaver ME, Jefferson LS. Effects of diabetes on protein synthesis in fast- and slow-twitch rat skeletal muscle. Am J Physiol. 1980;239(1):E88-95.

14. Ashford AJ, Pain VM. Effect of diabetes on the rates of synthesis and degradation of ribosomes in rat muscle and liver in vivo. J Biol Chem. 1986;261(9):4059-65.

15. Nader GA, Esser KA. Intracellular signaling specificity in skeletal muscle in response to different modes of exercise. J Appl Physiol (1985). 2001;90(5):1936-42

16. Carraro F, Stuart CA, HartI WH, Rosenblatt J, Wolfe RR. Effect of exercise and recovery on muscle protein synthesis in human subjects. Am J Physiol. 1990;259(4 Pt 1):E470-6.

17. Xie Z, Klionsky DJ. Autophagosome formation: core machinery and adaptations. Nat Cell Biol. 2007:9(10):1102-9.

18. Kraft C, Deplazes A, Sohrmann M, Peter M. Mature ribosomes are selectively degraded upon starvation by an autophagy pathway requiring the Ubp3p/Bre5p ubiquitin protease. Nat Cell Biol. 2008;10(5):602-10.

19. Sloan KE, Schneider C, Watkins NJ. Comparison of the yeast and human nuclear exosome complexes. Biochem Soc Trans. 2012;40(4):850-5.

20. Philp A, Schenk S, Perez-Schindler J, Hamilton DL, Breen L, Laverone E, Jeromson S, Phillips SM, Baar K. Rapamycin does not prevent increases in myofibrillar or mitochondrial protein synthesis following endurance exercise. J Physiol. 2015;593(18):4275-84.

\section{Submit your next manuscript to BioMed Central and we will help you at every step:}

- We accept pre-submission inquiries

- Our selector tool helps you to find the most relevant journal

- We provide round the clock customer support

- Convenient online submission

- Thorough peer review

- Inclusion in PubMed and all major indexing services

- Maximum visibility for your research

Submit your manuscript at www.biomedcentral.com/submit 